\title{
Broad emission lines for a negatively spinning black hole
}

\author{
T. Dauser ${ }^{1}$, J. Wilms ${ }^{1}$, C. S. Reynolds ${ }^{2}$, and L. W. Brenneman ${ }^{3}$ \\ ${ }^{1}$ Dr. Karl Remeis-Observatory and ECAP, Sternwartstr. 7, 96049 Bamberg, Germany \\ email: thomas.dauser@sternwarte.uni-erlangen.de \\ ${ }^{2}$ Department of Astronomy and Maryland Astronomy Center for Theory and Computation, \\ University of Maryland, College Park, MD 20742, USA \\ ${ }^{3}$ Harvard-Smithsonian C. for Astrophysics, 60 Garden Street, Cambridge, MA 02138, USA
}

\begin{abstract}
We present an extended scheme for the calculation of the profiles of emission lines from accretion disks around rotating black holes. The scheme includes disks with angular momenta which are parallel and antiparallel with respect to the black hole's angular momentum, as both configurations are assumed to be stable (King et al. 2005). Based on a Green's function approach, an arbitrary radius dependence of the disk emissivity and arbitrary limb darkening laws can be easily taken into account, while the amount of precomputed data is significantly reduced with respect to other available models. We discuss line shapes for such disks and present a code for modelling observational data with this scheme in X-ray data analysis programs. A detailed discussion will soon be presented in a forthcoming paper (Dauser et al. 2010).
\end{abstract}

Keywords. accretion, accretion disks, black hole physics, galaxies: nuclei, galaxies: active, lines: profiles

\section{How does a black hole get negative spin?}

Misalignments between the angular momenta of a black hole and its accretion disk are a prediction of stochastic evolution models for AGN (e.g., Volonteri et al. 2005). In galactic black holes, misalignments can be produced by the initial supernova kick (Brandt \& Podsiadlowski 1995). As shown by King et al. (2005), both parallel and antiparallel alignments of the disk and black hole angular momenta are stable configurations; misaligned discs will evolve to one of them. In fact, accretion onto rapidly-spinning retrograde black holes, where the angle between the angular momenta is greater than $90^{\circ}$, may be of some importance for understanding the properties of powerful radio-loud AGN. Garofalo (2009) argues that an accretion disk around a retrograde black hole is a particularly potent configuration for generating powerful jets, which might also explain the lack of radio-loud AGN (Garofalo et al. 2010).

\section{Line Profiles}

Since the shape of the observed emission line (e.g. at $6.4 \mathrm{keV}$ for a neutral $\mathrm{Fe} \mathrm{K} \alpha$ transition) depends on the spin of the black hole, $a$, and the emissivity (i.e., the intensity dependence on the radius) and inclination of the surrounding accretion disk, the diagnostic power of relativistic lines is very high, as they provide one of the most direct ways to probe the physics of the region of strong gravity close to the black hole.

In order to allow for a comparison of the pure frame-dragging effects, the inner edge of the accretion disk in the left part of Fig. 1 was set to $9 r_{\mathrm{g}}$ for all profiles, which is the smallest inner radius possible for an accretion disk around a maximally negatively rotating black hole. The major difference between the different spins is the relative strength 

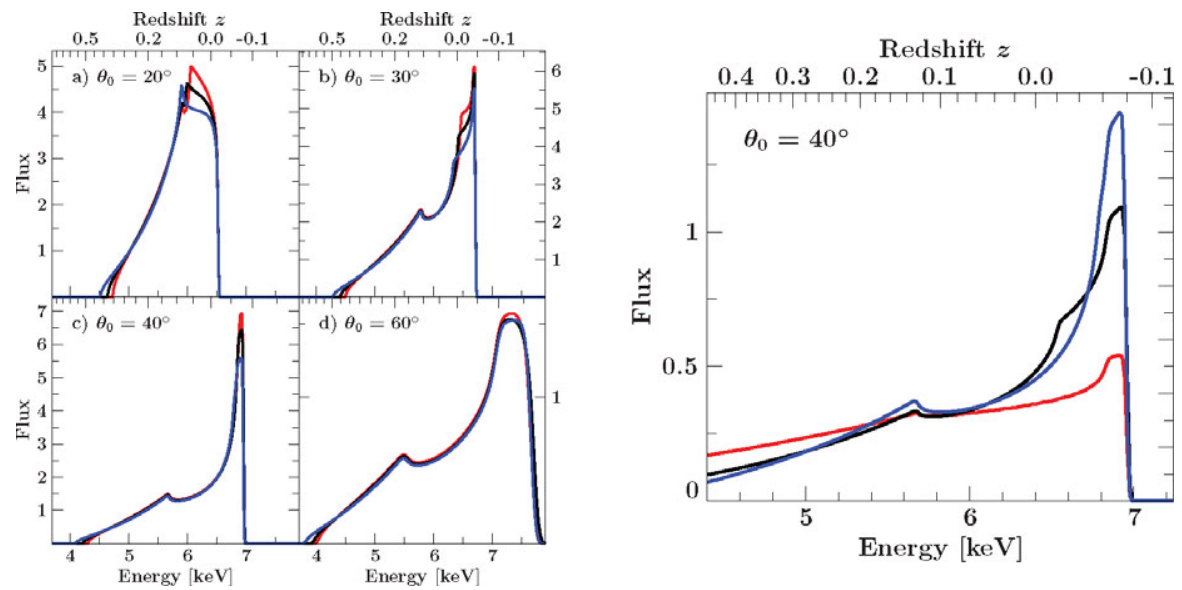

Figure 1. Line profiles for a relativistic iron line at $6.4 \mathrm{keV}$, an emissivity of $r^{-3}$ and under an inclination angle of $\theta_{o}$. The maximal spinning black hole $(a=+0.998)$ is drawn in red, the non-rotating $(a=0)$ in black and the line for maximal negative spin $(a=-0.998)$ in blue. Left: Accretion disk with an inner radius of $9 r_{\mathrm{g}}$. Right: Line for a disk extending down to the marginal stable orbit, which varies from $1.23 r_{\mathrm{g}}(a=+0.998)$ to $9 r_{\mathrm{g}}(a=-0.998)$.

of the core of the line to the red wing, which decreases with decreasing $a$. For this case of a large inner radius, the most significant differences in line shape are seen for low values of $\theta_{\mathrm{o}}$ while the red tails are virtually indistinguishable.

The more realistic case, where the disk extends down to the marginally stable orbit, is shown in the right part of Fig. 1. Since the inner edge of the disk is closer to the black hole for positively spinning black holes, more strongly redshifted photons emerge and therefore broader lines (especially for emissivities strongly peaked inwards).

\section{Data Analysis}

As none of the currently available line models like the kerrdisk (Brenneman \& Reynolds 2006) or the ky-family of models (Dovčiak et al. 2004) are valid for retrograde accretion disks, we implemented a model function, called relline, that can be added to data analysis software such as ISIS or XSPEC for spin $-0.998 \leqslant a \leqslant 0.998$ and inclination $0^{\circ} \leqslant \theta \leqslant 89^{\circ}$. Both, an additive and a convolution model are provided at www. sternwarte.uni-erlangen.de/research/relline/.

Acknowledgements: The research presented in these contributions was partially financed by the European Commission under grant ISN 215212.

\section{References}

Brandt N., \& Podsiadlowski P., 1995, MNRAS 274, 461

Brenneman L. W., \& Reynolds C. S., 2006, ApJ 652, 1028

Dauser T., Wilms J., Reynolds C. S., \& Brenneman L. W., 2010, MNRAS in press, arxive: 1007.4937

Dovčiak M., Karas V., \& Yaqoob T., 2004, ApJ Suppl. 153, 205

Garofalo D., 2009, ApJ 699, 400

Garofalo D., Evans D. A., \& Sambruna R. M., 2010, MNRAS 406, 975

King A. R., Lubow S. H., Ogilvie G. I., \& Pringle J. E., 2005, MNRAS 363, 49

Volonteri M., Madau P., Quataert E., \& Rees M. J., 2005, ApJ 620, 69 